\title{
Réhabilitation nutritionnelle à base des laits F75 et F100 de patients âgés de 6 à 59 mois en phase de stabilisation en milieu hospitalier à Niamey (Niger)
}

\author{
Sadou KANGAYE ${ }^{1 *}$, Kamaye MOUMOUNI ${ }^{2}$, Alido SOUMANA ${ }^{4}$ Alkassoum IBRAHIM $^{3}$, \\ Mamane OUSMAN ${ }^{4}$. Hassane MOUMOUNI ${ }^{3}$ et Hassimi SADOU ${ }^{1}$ \\ ${ }^{1}$ Laboratoire de Nutrition et Valorisation des Agro-Ressources. Faculté des Sciences et Techniques. Université \\ Abdou Moumouni. Niamey, Niger. \\ ${ }^{2}$ Service des urgences pédiatriques. Hôpital National de Niamey. \\ ${ }^{3}$ Faculté des Sciences de la Santé. Université Abdou Moumouni, BP : 131/125 Niamey, Niger. \\ ${ }^{4}$ Centre de Réhabilitation Nutritionnelle Interne. Service de Pédiatrie. Hôpital National de Lamordé. BP : \\ 11653 Niamey, Niger. \\ *Auteur correspondant; E-mail: kangayesadou@yahoo.com; Tel: (+227) 97250084
}

\section{RESUME}

En l'absence d'intervention diététique, tout état morbide entraînerait un affaiblissement des réserves énergétiques et un bilan azoté négatif. L'objectif de l'étude était de déterminer la pertinence d'une réhabilitation nutritionnelle systématique à base de laits F75 et F100 aux malades hospitalisés aux urgences pédiatriques. Il s'agit d'une étude descriptive transversale conduite du 16 janvier au 2 mai 2016 dans deux centres hospitaliers de référence de Niamey. 287 enfants âgés de 6 à 59 mois ont été concernés. Lorsqu'un enfant tombait malade, environ $67 \%$ des mères mettaient plus de 72 heures avant de recourir à l'hôpital. Ce retard dans la consultation aggravait significativement l'état nutritionnel de l'enfant $(\mathrm{p}<0,05)$. L'intervention diététique a amélioré significativement les paramètres cliniques comme l'état de conscience $(\mathrm{p}=0,012)$, la taille du foie $(\mathrm{p}=0,001)$ et la souplesse abdominale $(\mathrm{p}=0,001)$. Les enfants étaient restés à $87 \%$, en phase de stabilisation, entre 5 à 7 jours. La durée moyenne-était de 3,6 jours. Environ 56\% des sujets avaient eu un gain de poids moyen de 6 à $10 \mathrm{~g} / \mathrm{Kg} / \mathrm{j}$ à leur sortie de la phase de stabilisation et $86 \%$ des mères se sont déclarées satisfaites. Une réhabilitation nutritionnelle systématique des patients hospitalisés aux urgences pédiatriques s'avère donc indispensable.

(C) 2018 International Formulae Group. All rights reserved.

Mots clés: Urgences pédiatriques, malnutrition, diététique, gain de poids.

\section{F75 and F100 milks based nutritional rehabilitation of patients from 6 to 59 months at the stabilisation stage in two national hospitals of Niamey (Niger)}

\begin{abstract}
In the absence of dietary intervention, any morbid state would lead to a weakening of the energy reserves and a negative nitrogen balance. This study was aimed to determine the interest of nutritional rehabilitation based on F75 and F100 milks for all patients hospitalized in pediatric emergencies units in Niger. It is a descriptive cross-sectional study led from January 16 to May 2, 2016 in two reference hospitals in Niamey. 287 children from 6 to 59 months were involved. The weight / height ratio (P / T) was used as a criterion for assessing
\end{abstract}


nutritional status. Patients with severe acute malnutrition (MAS) have received F75 milk, patients with moderate malnutrition (MAM) and those without proven malnutrition, had received F100 milk. When a child failed ill, about $67 \%$ of mothers waited for more than 72 hours before taking him to the hospital. In consultation, this delay worsened significantly the nutritional status of the child ( $\mathrm{p}<0.05)$. The prevalence of general acute malnutrition (GAM) was 33\% in the age group between 6 to 12 months, $27 \%$ in the group from 13 to 24 months and $17 \%$ for those from 25 to 59 months. Children from 6 to 24 months were most at risk ( $p=$ $0.02)$. The dietary intervention improved significantly the clinical parameters such as the consciousness state $(\mathrm{p}$ $=0.012)$, the liver size $(p=0.001)$ and the abdominal flexibility $(p=0.001) .87 \%$ of the patients remained between 5 to 7 days in the stabilization phase. The average duration was 3.6 days. About $56 \%$ of the subjects had an average weight gain from 6 to $10 \mathrm{~g} / \mathrm{kg} /$ day when they left the stabilization phase. $86 \%$ of the mothers were satisfied. Therefore it is important to systematize nutritional rehabilitation for patients hospitalized in pediatric emergency units.

(C) 2018 International Formulae Group. All rights reserved.

Key words: Pediatric emergency, malnutrition, dietetic, weight gain.

\section{INTRODUCTION}

$\mathrm{Au}$ Niger, la pratique des soins pédiatriques actuels se caractérise par les seules applications diététiques recommandées par le protocole national de prise en charge intégrée de la malnutrition aiguë globale au Niger. Une des limites de ce protocole même dans ses versions révisées réside dans le fait qu'il ne propose de prise en charge diététique que pour les enfants malnutris sévères hospitalisés (MSP, 2005). Pourtant, le processus de la dénutrition est plus précoce et plus complexe que l'on pourrait y penser. En effet, l'organisme ne disposant pas de réserves protéiques et ne possédant de réserves glucidiques que pour quelques heures, toute réduction d'apports alimentaires associée ou non à une accélération du catabolisme protéique entraînerait une baisse des réserves énergétiques et une négativation du bilan d'azote (Peláez, 2013; Gouveia et Silva, 2017). Ce qui entraîne la mise en place de mécanismes d'adaptation physiologique indispensables pour assurer au cerveau des substrats énergétiques par les voies de la néoglucogenèse et de la cétogenèse. Ce processus serait plus prononcé chez un sujet malade lorsque la pathologie (aiguë ou chronique) occasionne une augmentation de la dépense énergétique, du catabolisme protéique et ou de la synthèse protéique (Joosten et al., 2008). En effet, toute utilisation excessive des protéines à des fins énergétiques entrainerait des altérations fonctionnelles responsables de morbi-mortalité chez les patients concernés (Schneider et al., 2004). Toutes les pathologies peuvent s'accompagner de dénutrition. Les âges extrêmes de la vie sont les plus touchés en raison des particularités physiologiques, d'une dépendance plus importante et du dépistage plus difficile (O'connor et al., 2004 ; Secker et al.,2007). Malheureusement, la dénutrition hospitalière reste encore largement sous-estimée, même si des études suggèrent une prévalence de l'ordre de 35 - 65\% en moyenne (Corish et al., 2000). Chez l'enfant hospitalisé, la dénutrition est souvent méconnue et l'apport énergétique ainsi que le suivi diététique sont insuffisants (Hankard et al., 2001). Pourtant, le risque de dénutrition apparaît dès qu'un enfant est malade et plus particulièrement chez l'enfant hospitalisé (Joosten et al., 2008, 2014 ; Pawelllek et al., 2008; Mehta et al., 2013). Elle pourrait également survenir au cours de l'hospitalisation même chez des sujets qui ne manifestaient aucun signe de dénutrition cliniquement décelable à leur admission (Sermet-Gaudelus et al., 2000 ; Rocha et al., 2006; Secker et al., 2007). Enfin, la dénutrition peut être insidieuse voire masquée par la prépondérance du tissu adipeux ou l'existence d'une rétention hydrosodée (Gouveia et Silva, 2017). Identifier les risques de dénutrition infantile à l'hôpital, évaluer son intensité et les risques de complications associés ainsi que l'efficacité de la thérapeutique de renutrition constituent donc un enjeu majeur de santé publique (Corish et al., 2000). De plus, si la maladie favorise et/ou aggrave la dénutrition, inversement la dénutrition aggrave la maladie, limite 
l'efficacité des traitements et augmente la durée d'hospitalisation (O'connor et al., 2004 ; Secker et al., 2007). La prise en charge nutritionnelle doit donc faire partie des soins de support au service de tout projet thérapeutique au même titre que la prévention et le traitement de la douleur ou des infections. Elle doit être encadrée par des professionnels ayant une compétence spécifique en concertation avec les autres soignants impliqués dans la prise en charge de la pathologie sous-jacente, et avec les parents de l'enfant (PNNS, 2010). Partant du constat de l'absence d'un protocole d'intervention diététique systématique chez les enfants dans les établissements de soins au Niger, la présente étude s'est donnée comme objectif de déterminer la pertinence d'une réhabilitation nutritionnelle à base de laits F75 et F100 (OMS, 2000; UNICEF, 2004) chez les patients âgés de 6 à 59 mois admis en urgence pédiatrique.

\section{MATERIEL ET METHODES Patients}

Il s'agit d'une étude descriptive transversale et analytique qui a concerné 287 enfants des deux sexes âgés de 6 à 59 mois admis en urgence pédiatrique. L'étude s'était déroulée du 16 janvier au 2 mai 2016 à l'Hôpital National de Niamey (HNN) et à l'Hôpital National Lamordé (HNL) sis aussi à Niamey. A leur arrivée, les enfants étaient reçus dans la salle d'accueil pour les procédures d'admission qui comprenaient, une ration de solution d'eau sucrée à $10 \%$, l'identification du patient, la recherche du motif de la consultation, la prise de la température, la pesée à l'aide d'une balance professionnelle de type SECA de $25 \pm 0,1 \mathrm{~kg}$, la détermination de la taille à l'aide d'une toise (Infantomètre modèle Unicef, précision de $0,1 \mathrm{~cm}$ ), et l'examen physique par le médecin de service. Les enfants ont été ensuite répartis en trois groupes selon la valeur du rapport poids pour la taille $(\mathrm{P} / \mathrm{T})$ (WHO, 2006):

- $\quad$ Sans malnutrition avérée : 02 ET $\leq$ $\mathrm{P} / \mathrm{T} \leq+2 \mathrm{ET} ;$ - 2 ET) ;
Malnutrition sévère : $\mathrm{P} / \mathrm{T} \leq-3 \mathrm{ET}$.

\section{Critères d'inclusion}

Tout enfant âgé de 6 à 59 mois hospitalisé en urgence pédiatrique pendant la période de l'étude et dont la mère a consenti participer à l'étude. Les sujets avec un état nutritionnel normal étaient aussi inclus.

Critères de non inclusion

Les patients obèses, ceux présentant des œdèmes et ceux de moins de 6 mois ou pesant moins de $4000 \mathrm{~g}$ de poids corporel n'ont pas été inclus dans l'étude. Ils étaient pris en charge suivant les directives du protocole national de la prise en charge intégrée de la malnutrition aiguë en vigueur au Niger.

\section{Considération éthique}

L'étude a été approuvée par le Comité national d'éthique et le Conseil scientifique académique de l'Université Abdou Moumouni et autorisée par les autorités des deux centres hospitaliers. Le protocole était conforme à la Déclaration d'Helsinki de 1975 révisée en 2008. La participation à l'étude était volontaire. Le but de l'étude a été expliqué aux parents et les consentements ont été obtenus. Une fiche individuelle de collecte des données a servi à l'enregistrement des informations obtenues à l'interview des mères, aux mesures anthropométriques et à l'observation clinique.

\section{Protocole opératoire}

Les aliments retenus pour la réhabilitation nutritionnelle étaient les laits F75 et F100 proposés en thérapie nutritionnelle dans les établissements de soins du Niger. Leur composition chimique est présentée dans le Tableau 1 (OMS, 2000; UNICEF, 2004).

Les patients malnutris sévères ont reçu une réhabilitation nutritionnelle à base de lait F75 fournissant $75 \mathrm{kcal} / 100 \mathrm{ml}$, les patients malnutris modérés et les patients sans malnutrition avérée à base du lait F100 apportant $100 \mathrm{kcal} / 100 \mathrm{ml}$. Les besoins énergétiques journaliers en $\mathrm{kcal} / \mathrm{kg}$ de poids corporel ont été estimés selon le Tableau 2. Pour le calcul des rations, des facteurs de correction ont été utilisés en fonction des symptômes sous-jacents comme indiqués dans 
le Tableau 3 (Müller-Schenker, 2004). Les rations journalières étaient prises sous forme de 8 repas par jour.

Le gain pondéral (GP) en $\mathrm{g} / \mathrm{kg} / \mathrm{jour}$ a été calculé selon la méthode de Ashworth et al. (2003) :

$\mathrm{GP}=\frac{\mathrm{P}_{3}-\mathrm{P}_{1}}{\mathrm{P}_{2} \times \mathrm{N}} \times 1000$

$\mathrm{P}_{3}$ : Poids à la sortie $(\mathrm{kg}) ; \mathrm{P}_{2}$ : Poids moyen $(\mathrm{kg}) ; \mathrm{P}_{1}=$ Poids à l'admission $(\mathrm{kg}) ; \mathrm{N}$ : Nombre de jours d'hospitalisation.

\section{Analyses statistiques}

L'analyse statistique a été réalisée à l'aide du logiciel SPSS version 22 (Chicago, Il, USA). Le test de $\mathrm{Chi}^{2}$ de Pearson a servi au test d'égalité entre deux proportions d'échantillons indépendants, substitué par le test exact de Fisher lorsque les conditions d'application le nécessitent. L'égalité entre deux moyennes d'échantillons indépendants a été déterminée par le test $\mathrm{t}$ de Student et lorsque les conditions d'application ne sont pas réunies, le test non paramétrique de Mann-Whitney était utilisé. La comparaison de plus de deux moyennes d'échantillons indépendants a été effectuée par le test non paramétrique de Kruskall-Wallis. Et le test des rangs de Wilcoxon a été utilisé pour l'analyse de la différence des moyennes d'avant et après intervention. Le seuil de signification choisi était de 0,05 pour toutes les analyses.

Tableau 1: Composition chimique des laits F75 et F100.

\begin{tabular}{lccccc}
\hline Eléments & Pour 100 g de produit sec & & \multicolumn{2}{c}{ Pour 1 litre de lait } \\
\cline { 2 - 3 } \cline { 5 - 6 } & Lait F-75 & F-100 & & Lait F-75 & F-100 \\
\hline \hline Energy & $446 \mathrm{kcal}$ & $520 \mathrm{kcal}$ & & $750 \mathrm{kcal}$ & $988 \mathrm{kcal}$ \\
Proteins & $0.9 \mathrm{~g}$ & $2.9 \mathrm{~g}$ & & $7 \mathrm{kcal}$ & $40 \mathrm{kcal}$ \\
Lipids & $31 \mathrm{~g}$ & $45 \mathrm{~g}$ & & $233 \mathrm{kcal}$ & $445 \mathrm{kcal}$ \\
Vit.A & $0,9 \mathrm{mg}$ & $800 \mu \mathrm{g}$ & & $1500 \mu \mathrm{g}$ & $1544 \mu \mathrm{g}$ \\
Vit.D & $18 \mu \mathrm{g}$ & $15 \mu \mathrm{g}$ & & $30 \mu \mathrm{g}$ & $29 \mu \mathrm{g}$ \\
Vit.E & $20 \mathrm{mg}$ & $20 \mathrm{mg}$ & & $34 \mathrm{mg}$ & $38,6 \mathrm{mg}$ \\
Vit.C & $59 \mathrm{mg}$ & $50 \mathrm{mg}$ & & $100 \mathrm{mg}$ & $96,5 \mathrm{mg}$ \\
Vit.B1 & $0,5 \mathrm{mg}$ & $0,5 \mathrm{mg}$ & & $0,8 \mathrm{mg}$ & $0,97 \mathrm{mg}$ \\
Vit.B2 & $1,2 \mathrm{mg}$ & $1,6 \mathrm{mg}$ & & $2,7 \mathrm{mg}$ & $3,1 \mathrm{mg}$ \\
Niacin & $5 \mathrm{mg}$ & $5 \mathrm{mg}$ & & $8,5 \mathrm{mg}$ & $9,7 \mathrm{mg}$ \\
Vit.B6 & $0,6 \mathrm{mg}$ & $0,6 \mathrm{mg}$ & & $1 \mathrm{mg}$ & $1,2 \mathrm{mg}$ \\
Folic.acid & $200 \mu \mathrm{g}$ & $200 \mu \mathrm{g}$ & & $340 \mu \mathrm{g}$ & $386 \mu \mathrm{g}$ \\
Vit.B12 & $1,6 \mu \mathrm{g}$ & $1,6 \mu \mathrm{g}$ & & $2,7 \mu \mathrm{g}$ & $3,1 \mu \mathrm{g}$ \\
Biotin & $60 \mu \mathrm{g}$ & $60 \mu \mathrm{g}$ & & $100 \mu \mathrm{g}$ & $116 \mu \mathrm{g}$ \\
Pantothenic acid & $3 \mathrm{mg}$ & $3 \mathrm{mg}$ & & $5,1 \mathrm{mg}$ & $5,8 \mathrm{mg}$ \\
Vit.k & $24 \mu \mathrm{g}$ & $15 \mu \mathrm{g}$ & & $40 \mu \mathrm{g}$ & $29 \mu \mathrm{g}$ \\
Sodium & $<87 \mathrm{mg}$ & $<290$ & & $<150 \mathrm{mg}$ & $<560 \mathrm{mg}$ \\
Calcium & $560 \mathrm{mg}$ & $300 \mathrm{mg}$ & & $950 \mathrm{mg}$ & $579 \mathrm{mg}$ \\
Phosphorus & $330 \mathrm{mg}$ & $300 \mathrm{mg}$ & & $560 \mathrm{mg}$ & $579 \mathrm{mg}$ \\
Magnesium & $50 \mathrm{mg}$ & $80 \mathrm{mg}$ & & $85 \mathrm{mg}$ & $154 \mathrm{mg}$ \\
Zinc & $12,2 \mathrm{mg}$ & $11 \mathrm{mg}$ & & $20,5 \mathrm{mg}$ & $21,2 \mathrm{mg}$ \\
Iodine & $100 \mu \mathrm{g}$ & $70 \mu \mathrm{g}$ & & $170 \mu \mathrm{g}$ & $135 \mu \mathrm{g}$ \\
Potassium & $775 \mathrm{mg}$ & $1100 \mathrm{mg}$ & & $1320 \mathrm{mg}$ & $2123 \mathrm{mg}$ \\
Copper & $1,7 \mathrm{mg}$ & $1,4 \mathrm{mg}$ & & $2,8 \mathrm{mg}$ & $2,7 \mathrm{mg}$ \\
Selenium & $30 \mu \mathrm{g}$ & $20 \mu \mathrm{g}$ & & $47 \mu \mathrm{g}$ & $38,6 \mu \mathrm{g}$ \\
Iron & $<0,3 \mathrm{mg}$ & $<0,2 \mathrm{mg}$ & & $<0,5 \mathrm{mg}$ & $<0,4 \mathrm{mg}$ \\
\hline
\end{tabular}


Tableau 2: Apports énergétiques des laits F100 et F75 en fonction de la tranche d'âge.

\begin{tabular}{lcc}
\hline Age (mois) & \multicolumn{2}{c}{$\mathbf{k c a l} / \mathbf{k g} / \mathbf{j}$} \\
\cline { 2 - 3 } & $\mathbf{F 1 0 0}$ & $\mathbf{F 7 5}$ \\
\hline \hline 6 & 110 & 83 \\
$7-9$ & 100 & 75 \\
1012 & 90 & 68 \\
$13-36$ & 1360 & 1020 \\
$37-59$ & 1830 & 1372 \\
\hline
\end{tabular}

Tableau 3: Pourcentage de correction des volumes des rations proposées en fonctions des symptômes.

\begin{tabular}{lccc}
\hline Symptômes & \multicolumn{3}{c}{ Pourcentage de correction des rations } \\
\cline { 2 - 4 } & $\begin{array}{l}\text { Sans malnutrition apparente } \\
\text { (F100) }\end{array}$ & $\begin{array}{c}\text { Malnutrition Modérée } \\
\text { (F100) }\end{array}$ & $\begin{array}{c}\text { Malnutrition sévère } \\
\text { (F75) }\end{array}$ \\
\hline \hline $\begin{array}{l}\text { Malformations } \\
\text { cardiaques }\end{array}$ & +40 & +20 & +20 \\
Paralysie cérébrale & -10 & -10 & -10 \\
Ventilation artificielle & -10 & -10 & -10 \\
Diarrhées & +50 & +20 & +20 \\
Vomissements & +50 & +20 & +20 \\
Sepsis & +30 & +30 & +20 \\
Fièvre & +20 & +10 & +10 \\
\hline
\end{tabular}

\section{RESULTATS}

Les provenances des enfants et les délais de consultation médicale des mères sont exprimés dans le Tableau 4. Les sujets admis en urgence pédiatrique provenaient à $74 \%$ de la Communauté Urbaine de Niamey (CUN) où sont situés les deux établissements de soins. La région de Tillabéry venait en seconde position avec $24,04 \%$ contre $1,96 \%$ pour le restant des localités. Cette région ceinture la CUN. La prévalence de la malnutrition aiguë globale (MAG) était d'environ $76 \%$ quel que soit la provenance dont $59 \%$ de malnutrition aiguë sévère (MAS) et $17 \%$ de malnutrition aigüe modérée (MAM), ( Tableau 4). Les principaux motifs de consultation des patients étaient un syndrome mal défini par la mère (Smd), la fièvre, la diarrhée, les Infections Respiratoires Aiguës (IRA), les vomissements avec respectivement $32,06 \%, 30,66 \%, 21,25 \%$, $9,06 \%$ et $6,975 \%$, (Tableau 9). Lorsqu'un enfant tombait malade, environ $67 \%$ des mères mettaient plus de $72 \mathrm{~h}$ avant de recourir à l'hôpital. Ce retard dans la consultation aggravait très significativement l'état nutritionnel de l'enfant $(p=0,003)$. Ainsi, sur les 218 enfants souffrant de MAG, 2/3 ont été enregistrés chez les mères ayant mis plus de 72 heures avant de consulter un médecin. Les raisons pour lesquelles les mères éprouvaient des difficultés à recourir à des soins de qualité étaient multiples allant de la méconnaissance des «signes de danger» de la maladie aux 
difficultés matérielles et financières pour effectuer le déplacement vers l'établissement de soins.-L'âge moyen des enfants hospitalisés aux urgences pédiatriques était $18,6 \pm 3$ mois et la médiane était de 15 mois avec 10,5 mois pour les garçons et 8,2 mois pour les filles. Le Tableau 5 présente la distribution de la malnutrition aiguë selon le sexe et la tranche d'âge. Sur les 287 enfants hospitalisés, la tranche d'âge 6 à 12 mois représentait $44 \%$, celle de 13 à 24 mois $35 \%$ et celle de 25 à 59 mois $21 \%$.

La prévalence de la malnutrition aiguë globale (MAG) était de $33 \%$ dans la tranche d'âge 6 à 12 mois, $27 \%$ dans celle de 13 à 24 mois et $17 \%$ dans celle de 25 à 59 mois. D'une manière générale, la prévalence de MAS était 3,5 fois plus élevée que celle de la MAM quelle que soit la tranche d'âge. L'âge de l'enfant était un facteur de risque d'hospitalisation $(p=0,005)$, la tranche d'âge la plus exposée était celle comprise entre 1324 mois $(p=0,044)$. L'âge était également un facteur majeur de risque de malnutrition chez l'enfant admis en urgence pédiatrique ( $\mathrm{p}$ $=0,001)$. Les enfants de 6-24 mois étaient plus exposés $(\mathrm{p}=0,02)$ et la tranche d'âge comprise entre 13 - 24 mois était la plus à risques $(\mathrm{p}=0,001)$.

Lorsqu'on considère le sexe, on constate qu'il y avait significativement plus de garçons hospitalisés $(57 \%)$ que de filles $(\mathrm{P}=$ $0,001)$. Les garçons étaient également plus exposés à la malnutrition $(\mathrm{p}=0,001)$ que les filles $(\mathrm{p}=0,065)$. La prévalence de la MAG était de $44 \%$ chez les garçons et $32 \%$ chez les filles.

Le sevrage débutait aux alentours de 6 mois avec l'introduction progressive d'une alimentation mixte constituée d'allaitements maternels et d'apports d'aliments de complément. Le Tableau 5 montre que certaines mères continuaient d'allaiter leurs enfants jusqu'à 24 mois. Le taux moyen d'allaitement au sein était de $89 \%$ dans la tranche d'âge 6 à 12 mois, il baissait significativement à $70 \%(\mathrm{p}=0,000)$ dans la tranche d'âge de 13 à 24 mois. Presque tous les garçons $(98,6 \%)$ et $76 \%$ des filles de 6 à 12 mois continuaient d'être allaités au sein.
Chez les enfants de 13 à 24 mois, 75\% des garçons et $63 \%$ des filles recevaient un allaitement maternel. Les garçons étaient plus allaités au sein que les filles $(\mathrm{p}=0,003)$ et leur allaitement durait plus longtemps que celui des filles $(p=0,001)$. Chez les enfants allaités au sein (Tableau 4), la prévalence de la MAS et de la MAM était respectivement de $59 \%$ et $17 \%$ dans la tranche d'âge de 6 à 12 mois contre $55 \%$ et $20 \%$ dans celle de 13 à 24 mois. Ainsi, si le taux global de la malnutrition semble uniforme entre les deux groupes, le degré de sévérité paraissait plus prononcé chez les 6 à 12 mois. La tranche d'âge de 6 mois à 24 mois était une période critique de transition où l'enfant passe progressivement d'une alimentation exclusive au sein à une alimentation adulte exclusive. D'où toute l'importance de centrer davantage les interventions de nutrition sur les stratégies de renforcement des capacités des mères en matière d'Alimentation du Nourrisson et du Jeune Enfant.

\section{Résultats de la supplémentation des sujets}

Le Tableau 6 montre la répartition des 287 enfants selon les facteurs de correction et le degré d'acceptation des rations de laits. Lorsqu'un enfant souffrait de plusieurs symptômes, le signe dominant était utilisé comme principal facteur de correction de la ration lactée. Il s'ensuivait que la fièvre représentait le principal facteur de correction $(51 \%)$ des rations de lait, suivie de la diarrhée $(43 \%)$ et des vomissements (4\%). L'acceptation des rations de lait a été bonne à $54 \%$, moyenne à $36 \%$ et médiocre avec nécessité de pose de sonde nasogastrique à $10 \%$. Cette autonomie des prises alimentaires prouvait que malgré leur état de santé les sujets ont conservé une certaine avidité pour les aliments.

\section{Indicateurs de la pertinence de l'intervention diététique}

Le Tableau 7 montre l'évolution des paramètres cliniques entre l'admission et la fin de la phase de stabilisation. Aucune complication n'a été observée. Au contraire, les états cliniques des sujets s'étaient 
améliorés de façon significative. Ainsi, $32,75 \%$ des patients étaient inconscient, $19,86 \%$ avaient une taille de foie anormale et $10,10 \%$ avaient une flatulence à l'admission. A la fin de la phase de stabilisation ces proportions étaient tombées à $1,04 \%(\mathrm{p}=0,012), 3,13 \%(\mathrm{p}=0,001)$ et $2,09 \%(\mathrm{p}=0,001)$ respectivement. Les fréquences de selles étaient supérieures à 3 pour $52,61 \%$ des patients à leur arrivée et le taux a chutée à $1,39 \%$. Cependant, aucune différence statistiquement significative n'a été observée pour ces fréquences de selles ( $\mathrm{p}$ $=0,201)$. Les taux de récupération des paramètres cliniques étaient pour l'état de conscience, la taille du foie, les fréquences des selles et les flatulences respectivement de $96,8 \%, 80,7 \%, 97,3 \%$ et $79,3 \%$. Il est important de noter que l'intervention diététique n'a provoqué aucun cas diarrhée.

\section{Impact de l'intervention diététique sur l'issue de l'hospitalisation}

Le Tableau 8 présente le gain pondéral, la durée d'hospitalisation et le niveau de satisfaction des mères. A la sortie de la phase de stabilisation, la baisse pondérale a été stoppée chez tous les patients, mieux, $60 \%$ ont eu des gains pondéraux moyens allant de 1 à plus de $11 \mathrm{~g} / \mathrm{Kg} / \mathrm{j}$. Environ $56 \%$ ont eu un gain de poids moyen de 6 à $10 \mathrm{~g} / \mathrm{Kg} / \mathrm{j}$ dont $33 \%$ d'enfants malnutris sévères, 9,6\% d'enfants malnutris modérés et $13,2 \%$ d'enfants non malnutris avérés. Le fait qu'une forte proportion des sujets ne prenait pas de poids $(39 \%)$, pour toutes les tranches nutritionnelles considérées, peut être mis en rapport avec l'objectif du traitement diététique dans cette première phase d'hospitalisation qui était d'aboutir à une stabilisation métabolique et non à une restauration pondérale. Les enfants sont restés à $87 \%$ en phase de stabilisation entre 5 à 7 jours avec $51,2 \%$ pour les malnutris sévères, $15 \%$ pour les modérés et $21 \%$ non malnutris avérés. La durée moyenne de la phase de stabilisation était de 3,6 jours. Les mères se sont déclarées satisfaites de l'intervention diététique à $86 \%$. Parmi les $14 \%$ de mères insatisfaites on note $8 \%$ de mères d'enfants malnutris sévères, $3,4 \%$ de mères de non malnutris avérés et $2,4 \%$ de mères de malnutris modérés. Les raisons invoquées ne sont pas liées à l'intervention diététique mais essentiellement au déficit de communication dans les unités d'accueil et à la lourdeur des procédures d'admission.

Tableau 4 : Impacts de la provenance, des délais de consultation médicale et du taux d'allaitement au sein sur le statut nutritionnel des patients âgés de 6 à 24 mois $(\mathrm{N}=183)$.

\begin{tabular}{|c|c|c|c|c|c|c|c|c|}
\hline \multirow{2}{*}{ Statut nutritionnel } & \multicolumn{2}{|c|}{$\begin{array}{c}\text { Provenance } \\
(\mathrm{N}=287)\end{array}$} & \multicolumn{4}{|c|}{$\begin{array}{l}\text { Délai de consultation } \\
\qquad(\mathrm{N}=\mathbf{2 8 7})\end{array}$} & \multicolumn{2}{|c|}{$\begin{array}{l}\text { Allaitement au sein } \\
\text { (en mois) }\end{array}$} \\
\hline & Niamey & *Autres & $24 h$ & $48 \mathrm{~h}$ & $72 \mathrm{~h}$ & $+72 \mathrm{~h}$ & $06-12$ & $13-24$ \\
\hline \multicolumn{9}{|c|}{$\mathrm{N}(\%)$} \\
\hline $\begin{array}{l}\text { Sans malnutrition } \\
\text { apparente }\end{array}$ & $51(17,7)$ & $18(6,2)$ & $6(2)$ & $11(3,8)$ & $6(2)$ & $46(16)$ & $27(24)$ & $18(25)$ \\
\hline $\begin{array}{l}\text { Malnutrition } \\
\text { modérée }\end{array}$ & $36(12,5)$ & $13(4,5)$ & $4(1,3)$ & $7(2,4)$ & $4(1)$ & $33(11)$ & $19(17)$ & $14(20)$ \\
\hline Malnutrition sévère & $125(43,5)$ & $44(15,3)$ & $16(5,5)$ & $26(9)$ & $\begin{array}{l}14 \\
(5)\end{array}$ & $\begin{array}{l}114 \\
(40)\end{array}$ & $66(59)$ & $39(55)$ \\
\hline Total & $212(73,8)$ & $75(26)$ & $26(9)$ & $44(15)$ & $24(8)$ & 193(67) & $\begin{array}{c}112 \\
(100)\end{array}$ & $71(100)$ \\
\hline
\end{tabular}

* Autres : Tillabéry (69 soit 24,04\%) et Doutchi 2, Mali 1, Burkina 2, Magaria 1 (soit au total 1,96\%) 
Tableau 5 : Prévalence de la malnutrition aigüe et taux l'allaitement au sein selon le sexe et la tranche d'âge des patients.

\begin{tabular}{clccccc}
\hline $\begin{array}{c}\text { Groupe d'âge } \\
\text { (mois) }\end{array}$ & Sexe & Total & $\begin{array}{c}\text { Malnutris } \\
\text { sévère }\end{array}$ & $\begin{array}{c}\text { Malnutris } \\
\text { modéré }\end{array}$ & Normonutris & $\begin{array}{c}\text { Allaitement } \\
\text { au sein }\end{array}$ \\
\hline \hline \multirow{2}{*}{$6-12$} & Garçons & $72(25,08)$ & $43(15)$ & $12(4,1)$ & $17(6)$ & $71(98,6)$ \\
& Filles & $54(18,8)$ & $32(11,1)$ & $9(3,1)$ & $13(4,5)$ & $41(76)$ \\
& Combinés & $126(43,9)$ & $75(26,1)$ & $21(7,3)$ & $30(10,5)$ & $112(89)$ \\
& Garçons & $59(20,5)$ & $35(12)$ & $10(3,4)$ & $14(4,8)$ & $44(74,5)$ \\
$13-24$ & Filles & $43(14,9)$ & $26(9)$ & $7(2,4)$ & $10(3,4)$ & $27(63)$ \\
& Combinés & $102(35,4)$ & $61(21)$ & $17(5,9)$ & $24(8,3)$ & $71(69,6)$ \\
& Garçons & $34(12)$ & $20(7)$ & $6(2)$ & $8(2,7)$ & \\
$25-59$ & Filles & $25(8,7)$ & $15(5,2)$ & $4(1,3)$ & $6(2)$ & \\
& Combinés & $59(20,7)$ & $35(12,2)$ & $10(3,4)$ & $14(4,7)$ & \\
\hline
\end{tabular}

Tableau 6: Répartition des sujets selon les facteurs de correction de la ration et le degré d'acceptation des laits $(\mathrm{N}=287)$.

\begin{tabular}{lcclcc}
\hline Symptôme & $\begin{array}{c}\text { Indice de correction du } \\
\text { volume de lait (\%) }\end{array}$ & $\mathbf{N}(\%)$ & \multicolumn{2}{l}{ Acceptation de la ration } & \\
\cline { 4 - 5 } & +20 & $147(51)$ & Bonne & $4 / 4$ & Qualité \\
\hline Fièvres & +50 & $123(43)$ & Moyenne & $3 / 4$ & $102(36)$ \\
Diarrhées & +50 & $12(4)$ & Médiocre & $2 / 4$ & $29(10)$ \\
Vomissements & 20 & $5(1,7)$ & Nulle & $-2 / 4$ & $0(0 \%)$ \\
Ventilations & & & & & \\
artificielles & & & & & \\
\hline
\end{tabular}

Tableau 7: Evolution des paramètres cliniques au cours de la phase de stabilisation.

\begin{tabular}{lcccc}
\hline $\begin{array}{l}\text { Paramètres cliniques (pré et post } \\
\text { examen) }\end{array}$ & Admission & Sortie & $\begin{array}{c}\text { *Taux de } \\
\text { récupération (\%) }\end{array}$ & P-Value \\
\hline \hline Etat de conscience anormale & $94(32,75)$ & $3(1.04)$ & 96,8 & 0,012 \\
Taille du foie démesurée & $57(19,86)$ & $9(3,13)$ & 80,7 & 0,0001 \\
Fréquences des selles & $151(52,61)$ & $4(1,39)$ & 97,3 & 0,201 \\
Flatulences abdominales & $29(10,10)$ & $6(2,09)$ & 79,3 & 0,0001 \\
\hline
\end{tabular}

* $\operatorname{Tr}($ Taux de récupération $)=\mathrm{N}$ (admission) $-\mathrm{N}($ sortie $) / \mathrm{N}($ admission $)$ x $100=\mathrm{x} \%$. 
Tableau 8: Gain pondéral, durée du séjour et niveau de satisfaction des mères à l'issue de l'hospitalisation.

\begin{tabular}{|c|c|c|c|c|c|c|c|c|}
\hline \multirow{2}{*}{$\begin{array}{c}\text { Statut } \\
\text { nutritionnel }\end{array}$} & \multicolumn{4}{|c|}{$\begin{array}{l}\text { Gains de poids moyen } \\
\text { g/Kg/J }(N=279)\end{array}$} & \multicolumn{2}{|c|}{$\begin{array}{l}\text { Durée du séjour } \\
\text { (jour) }(\mathbf{N}=\mathbf{2 8 7})\end{array}$} & \multicolumn{2}{|c|}{$\begin{array}{l}\text { Satisfaction des } \\
\text { mères }(\mathrm{N}=287)\end{array}$} \\
\hline & 0 & $1-5$ & $6-10$ & $\geq 11$ & $5-7$ & 8 et plus & Oui & Non \\
\hline $\begin{array}{l}\text { Sans } \\
\text { malnutrition } \\
\text { apparente }\end{array}$ & $26(9,3)$ & $2(0,7)$ & $37(13,2)$ & $1(0,3)$ & $60(21)$ & $9(3,1)$ & $59(20,5)$ & $10(3,4)$ \\
\hline $\begin{array}{l}\text { Malnutrition } \\
\text { modérée }\end{array}$ & $19(6,8)$ & $1(0,3)$ & $27(9,6)$ & $1(0,3)$ & $43(15)$ & $6(2,09)$ & $42(14,6)$ & $7(2,4)$ \\
\hline $\begin{array}{l}\text { Malnutrition } \\
\text { sévère }\end{array}$ & $65(15)$ & $5(1,7)$ & $92(33)$ & $3(1)$ & $147(51,2)$ & $22(7,6)$ & $146(50,8)$ & $23(8)$ \\
\hline Total & $110(39,4)$ & $8(2,7)$ & $156(55,7)$ & $5(1,6)$ & $250(87,2)$ & $37(12,8)$ & 247(86) & $40(13,8)$ \\
\hline
\end{tabular}

$\mathrm{Nb}: 8$ fiches n'ont pas retenus pour insuffisance dans le remplissage.

Tableau 9 : Fréquence des motifs de consultation.

\begin{tabular}{|c|c|}
\hline Symptômes & Fréquences $\mathrm{N}(\%)$ \\
\hline Fièvre & $88(30,66)$ \\
\hline Diarrhée & $61(21,25)$ \\
\hline IRA & $26(9,06)$ \\
\hline Vomissements & $20(6,97)$ \\
\hline *Smd & $92(32,06)$ \\
\hline
\end{tabular}

\section{DISCUSSION}

Les sujets admis en urgence pédiatrique proviennent essentiellement des zones urbaines et suburbaines de la capitale Niamey comme cela était le cas au Burkina Faso (Ouédraogo, 2012) et au Mali (Barry, 2008). Environ $67 \%$ des mères mettent plus de 3 jours avant de se décider à consulter un médecin. Cette longue attente affecte négativement l'état nutritionnel de l'enfant. $\mathrm{Au}$ Burkina Faso, le délai maximum de réponse des mères était de $24 \mathrm{H}$ (Ouédraogo, 2012). Au cours de la présente étude, seule, $2 \%$ des mères consultent un médecin dans les $24 \mathrm{~h}$. Les raisons pour lesquelles les mères éprouvent des difficultés à recourir à des soins de qualité sont multiples allant de la méconnaissance des «signes de danger » de la maladie aux difficultés matérielles et financières pour effectuer le déplacement vers l'établissement de soins.

Dans les pays développés, la prévalence de la malnutrition aiguë, à l'hospitalisation, varie de $6,1 \%$ à $19 \%$ (Sermet-Gaudelus et al., 2013). Dans des pays émergents comme le Brésil et la Turquie, la prévalence de la malnutrition hospitalière aiguë à l'admission est plus élevée, allant de $33,8 \%$ à 52,4\% (Rocha et al., 2006 ; Oztürk et al., 2003 ; Gouveia et al., 2016). Chez les 287 enfants hospitalisés, la prévalence de la MAG atteint le chiffre alarmant de $76 \%$. De plus, le 
ratio MAS sur MAM qui était de 0,52 dans la population infantile (INS, 2013) est inversé et passe à 3,5 en milieu hospitalier. Ainsi, chez l'enfant malade, non seulement on assiste à une explosion de la MAG mais on observe aussi une prédominance inquiétante de la MAS. La prise en charge de la malnutrition chez l'enfant hospitalisé mérite donc une attention toute particulière. Surtout quand on sait que la malnutrition hospitalière contribue à la morbidité et à la mortalité des enfants mais aussi à celles des adultes, car elle affaiblit le système immunitaire, augmente le risque d'infections, retarde la guérison des plaies, réduit les fonctions du tractus gastrointestinal, augmente la dépendance à la ventilation mécanique, augmente la durée du séjour hospitalier et augmente les coûts hospitaliers (Correia et Waitzberg, 2003). La prise en charge diététique doit donc faire partie de tout projet thérapeutique au même titre que le traitement des infections sousjacentes.

Au cours de la présente étude, $44 \%$ des enfants souffrant de MAG appartiennent à la tranche d'âge 6 à 12 mois avec les enfants de $12-24$ mois, on a $79 \%$ de l'effectif. Cette forte vulnérabilité des 6 à 24 mois a déjà été rapportée au Bénin où, la malnutrition touchait préférentiellement, la classe de 12-23 mois et ceci de manière plus significative pour le retard statural, $27 \%$ vs $17,5 \%$ pour les autres classes (Main, 1975 ; Sinnaeve, 2006 ; Ibrahim, 2016). Il s'agit d'une période critique pour l'enfant au cours de laquelle il passe progressivement d'un allaitement au sein exclusif à une alimentation adulte. En effet, au Niger, la plupart des mères commencent l'introduction des aliments de compléments à leurs enfants à 6 mois et environ $62 \%$ poursuivent cette alimentation mixte jusqu'à 24 mois (Wuehler et Biga, 2011).

Sur les 287 enfants hospitalisés, les garçons représentent $57 \%$, ce qui correspond à un sexe ratio de 1,33. Entre janvier 2008 et juillet 2009, sur 426 enfant hospitalisés dans le même service, les garçons représentaient 55,3\% (Madec et al., 2010). La prévalence de la MAG est de $43,4 \%$ chez les garçons dont
$34 \%$ de MAS et $32,4 \%$ chez les filles avec $25 \%$ de MAS. Au niveau national, la prévalence de la MAG est de 19,2\% chez les garçons et $16,2 \%$ chez les filles et la MAS représente $6,9 \%$ et $5,4 \%$ respectivement (INS, 2013). La malnutrition aiguë constitue un problème majeur de Santé Publique au Niger chez les enfants de moins de 5 ans et selon notre étude sa prévalence est plus prononcée chez les garçons que chez les filles $(p=0,04)$. Le sexe ratio entre les garçons MAG versus les filles MAG est de 1,14 dans la population infantile de moins de 5 ans, il passe à 1,28 chez les enfants hospitalisés. Cette prédominance des garçons par rapport aux filles dans notre série s'accorde aux résultats d'Ouagadougou au Burkina Faso qui rapportent $47,4 \%$ pour les garçons vs $46,5 \%$ de filles émaciées (Yugbare/Ouedraogo et al., 2016). Toutefois, selon Ousmane (2012) au Burkina Faso, ce sont plutôt les filles qui seraient plus sensibles à la malnutrition avec $51,7 \%$ pour le sexe féminin et $48,3 \%$ pour le sexe masculin.

F75 et F100 sont des laits spécialement formulés qui sont utilisés dans les établissements hospitaliers pour traiter la malnutrition aiguë sévère (Deen et al., 2003 ; UNICEF, 2014). Nous avons choisi ces deux formulations pour une réhabilitation nutritionnelle systématiques des enfants admis en urgences pédiatriques au cours de la phase de stabilisation (Briend, 2003). F75 a été administré aux enfants malnutris sévères et F100 aux malnutris modérés et aux enfants sans malnutrition apparente. Le symptôme principal dont souffre l'enfant a été utilisé pour corriger les rations journalières (MüllerSchenker, 2004). La fièvre représente le principal facteur de correction des rations de lait $(51 \%)$, suivie des diarrhées $(43 \%)$, des vomissements $(4 \%)$. Ces signes cliniques constituent aussi, pour la plupart des mères, les causes de consultation médicale pour leurs enfants (Koné, 2008). Aucun incident clinique lié à l'intervention diététique n'a été enregistré. Au contraire, des paramètres cliniques tels que l'état de conscience, la taille 
$\mathrm{du}$ foie et la souplesse abdominale se sont améliorés de façon significative.

Le gain pondéral est l'indicateur le plus utilisée pour apprécier l'efficacité d'une réhabilitation nutritionnelle. $56 \%$ des sujets ont eu un gain de poids de $6-10 \mathrm{~g} / \mathrm{kg} / \mathrm{j}$ dont $33 \%$ enfants malnutris sévère, $13,2 \%$ enfants non malnutris avérés et $9,6 \%$ malnutris modérés. L'OMS (2000) recommande pour une bonne réhabilitation nutritionnelle des enfants malnutris, un gain pondéral journalier de $20 \mathrm{~g} / \mathrm{kg}$ par jour. Cependant, elle estime qu'un gain pondéral journalier de 10 à $15 \mathrm{~g} / \mathrm{kg}$ par jour est acceptable. Le gain pondéral journalier obtenu au cours de la présente étude est relativement bas. Il faut cependant rappeler que l'objectif de la stratégie diététique au cours de cette phase de stabilisation n'est pas le gain pondéral qui était surtout attendu au cours de la phase de réhabilitation nutritionnelle. Il s'agit plutôt d'obtenir une stabilisation pondérale chez les sujets dénutris et d'empêcher une perte pondérale chez les autres. Il s'agit de corriger ou de prévenir toute malnutrition hospitalière. A ce titre, l'objectif a été largement atteint car tous les enfants ont eu un poids stabilisé et $66 \%$ remonté. Il y a lieu donc de standardiser une récupération nutritionnelle pour tous les enfants malades. Une amélioration de l'état nutritionnel des enfants passe par une diversification alimentaire bien conduite mais nécessite encore de nombreux efforts pour une meilleure information des mères (Yugbare/Ouedraogo et al., 2016). Par ailleurs, une utilisation des fortifiants des aliments localement disponibles telle que la spiruline proposée par certains auteurs est envisageable (Mamadou et al., 2014; Branger et al., 2003; Simpore et al., 2006 ; Sall et al., 2009). Ce qui permettrait également en accord avec Aliou (2012) de promouvoir l'économie dans cette zone ouest africaine essentiellement agricole. Elle permettra aussi vers une exploitation plus judicieuse des produits de l'élevage en remplacement d'une production de prestige actuellement prédominante (Youssao Abdou Karim et al., 2013).

Chez des patients marasmiques de 6 à 59 mois, la durée moyenne de la phase de stabilisation rapportée était 4 jours. Elle peut aller de 5 à 7 jours en cas de kwashiorkor, de kwashiokor-marasme sinon plus en cas de d'une gastroentérite associée au paludisme (Nguefack et al., 2015). Au cours de la présente étude, les enfants sont restés à $87 \%$ en phase de stabilisation entre 5 à 7 ; la durée moyenne est de 3,6 j. La durée moyenne de la phase de stabilisation, avant l'intervention diététique, était de 11 jours, 3 fois plus long (Halidou, 2011). Un long séjour à l'hôpital n'est pas sans inconvénients, il peut être coûteux pour les parents et exposer l'enfant à des risques d'infections (Correia et Waitzberg, 2013). A l'issue de la phase de stabilisation, les enfants malnutris sont réorientés vers les centres extérieurs de récupération nutritionnelle conformément aux directives du Protocole national de prise en charge intégrée de la malnutrition. $87 \%$ des mères se sont déclarées satisfaites de l'intervention diététique et de la rémission rapide de leurs enfants. Les $16 \%$ de mères insatisfaites ont mis en cause le défaut de communication et des relations exécrables entre soignants et soignés.

\section{Conclusion}

Les mères mettent un long délai de consultation qui impacte négativement l'état nutritionnel de leurs enfants. Dans notre série, l'intervention diététique a permis, chez les patients en phase de stabilisation, un bon maintien de l'état de conscience, une régularisation du transit intestinal, une durée de séjours raisonnable et une satisfaction des mères. Les rations n'ont occasionné ni de distension abdominale, ni de démesure du foie. Une réhabilitation nutritionnelle s'impose chez l'enfant admis en urgence pédiatrique en complément des soins médicaux. L'étude s'étant déroulée dans deux hôpitaux nationaux de référence, pour la compléter, il est recommandable de la 
répliquer dans les Centres Hospitaliers Régionaux et mieux avec des aliments locaux.

\section{CONFLITS D'INTERETS}

Il n'existe aucun conflit d'intérêt pour le présent article.

\section{CONTRIBUTIONS DES AUTEURS}

SK a participé à la réalisation des travaux de cette recherche et est titulaire de l'article, KM, AS, MO l'encadrement technique sur le terrain, AI et HM conseils externes de la recherche, et HS a été le directeur de la recherche. Tous ont lu et approuvé la version finale du manuscrit.

\section{REMERCIEMENTS}

Nos sincères remerciements vont au Projet d'Appui Institutionnel de la Coopération Technique Belge (CTB) pour avoir financé ce travail, le Fonds des Nations Unies pour l'Enfance (UNICEF) pour l'accompagnement en Laits F75 et F100, les personnels des Hôpitaux Nationaux de Niamey et Lamordé pour la collecte de données, le personnel de l'Institut National de la Statistique pour le traitement des données.

\section{REFERENCES}

Ashworth A, Khanum S, Jackson A, Schofield C. 2003. Guidelines for the inpatient treatment of severely malnourished children. World Health Organization. PDF

http://www.Who.int,guide_inpatient_te $\mathrm{xt}$

Aliou M, Dansou K, Charles A, Paul R, Thomas W. 2012. Effects of farmers' practices of fertilizer application and land use types on subsequent maize yield and nutrient uptake in central Benin. Int. J. Biol. Chem. Sci., 6(1): 365-378.

DOI : http://dx.doi.org/10.4314/ijbcs.v6i1.32.

Barry B. 2009. Evaluation de la Prise en Charge de la malnutrition aiguë sévère dans le service de pédiatrie de l'hôpital de Gao, (Mali). Thèse de médecine, Université de Bamako (FMPO), Bamako, p.74.
Branger B, Cadudal J, delobel, M Ouoba H, Yameogo P, Ouedraogo D. 2003. Spirulina as food supplement in case of infant malnutrition in Burkina- Faso. Arch Pediatr, 10(5): 424-431.

Briend A. 2003. Prise en charge de la malnutrition sévère de l'enfant en milieu tropical. Med. Trop., 63(4-5): 527- 532. DOI : http://researchgate.net, publication

Correia MI, Waitzberg DL. 2003. The impact of malnutrition on morbidity, mortality, length of hospital stay and costs evaluated through a multivariate model analysis. Clin Nutr., 22(3): 235-239. DOI: http://www.ncbinlm.nih.gov, pubmed

Corish CA, Kennedy NP. 2000. Proteinenergy under nutrition in hospital inpatients. Br. J. Nutr., 83: 575-591. DOI: http://www.ncbinlm.nih.gov, pubmed

Deen JL, Funk M, Guevara VC, Saloojee H, Doe JY, Palmer A, et al. 2003. Implementation of WHO guidelines on management of severe malnutrition in hospitals in Africa. Bull. World Health Organ., 81(4): 237-245. DOI: http://www.ncbinlm.nih.gov, pubmed

Gouveia MAC, Silva GAP. 2017. Hospital malnutrition in pediatric patients: a review. Ann. Nutr. Disord. Ther., 4(2): id1042. DOI: http://austinpublishing. Group.Com. PDF

Halidou M. 2011. Evaluation de la prise en charge des enfants malnutris sévères au Centre de Réhabilitation nutritionnelle Interne- CRENI- de l'Hôpital Lamordé (HNL). Thèse de médecine, Université Abdou Moumouni-Niamey (UAM), Niger, p.86.

Hankard R, Bloch J, Martin P, Randrianasolo H, Bannier MF, Machinot S, Cézard JP. 2001. Nutritional status and risk in hospitalized children. Arch. Pediatr., 8(11): 1203-1208. DOI: http://www.ncbinlm.nih.gov, pubmed

INS (Institut National de la Statistique) et ICF International. 2013. Enquête Démographique et de Santé et à 
Indicateurs Multiples du Niger 2012. INS et ICF International. Calverton, Maryland, USA. DOI : https://dhsprogram.com/pubs/pdf/FR27 7/FR277.pdf

Joosten KFM, Hulst JM. 2008. Prevalence of malnutrition in pediatric hospital patients. Curr. Opin. Pediatr. ; 20: 590596. PDF

DOI: http://www.ncbinlm.nih.gov, pubmed

Joosten KFM, Hulst JM. 2014. Nutritional screening tools for hospitalized children: methodological considerations. Clin. Nutr., 33: 1-5. DOI:

http://wwwstuurgroepondervoeding.nl

Madec Y, Germanaud D, Moya-Alvarez V, Alkassoum W, Issa A, Amadou M, Tchiombiano S, Pizzocolo C, Huber F, Diallo S, Abdoulaye-Mamadou R. 2011. HIV Prevalence and Impact on Renutrition in Children Hospitalised for Severe Malnutrition in Niger: An Argument for More Systematic Screening. Plos One., 6(7): e22787. DOI:

http://www.Journals.plos.org.plosone,article

Mamadou B, Moussa O, Seydou S, Innocent P. 2014. Intérêt thérapeutique de la spiruline chez l'homme: revue générale. DOI: http:/dx.doi.org/10.4314/ijbcs.v8i6.33

Mehta NM, Corkins MR, Lyman B, Malone A, Goday PS, Carney LN, et al. 2013. Defining pediatric malnutrition: a paradigm shift toward etiologyrelated definitions. J. Parenter Enteral Nutr., 37: 460-481. DOI: http://www.ncbinlm.nih.gov, pubmed

Müller-Schenker B. 2004. Alimentation entérale : Indications - application pratique - risques. Paediatrica, 15(5) : $22-25$.

DOI: http://www.swiss.peadiatrics.org, node

MSP (Ministère de la Santé Publique). 2005. Protocole national de prise en charge de la malnutrition. MSP / UNICEF / OMS /HKI Edition. Niger 2005. 69 p. Disponible sur: http://www.who.int/hac/crises/ner/back
ground/UNICEF_Protocole_national_N iger_final.pdf. Consulté le 30 juin 2017.

Nguefack F, Adjahoung CA, Keugoung B, Kamgaing N, Dongmo R. 2015. Prise en charge hospitalière de la malnutrition aigüe sévère chez l'enfant avec des préparations locales alternatives aux F-75 et F-100: résultats et défis. Pan. Afr. Med. J., 21: 329. DOI: https://www.panafrican-medjournal.com

O' Connor J, Youde LS, Allen JR, Hanson RM, Baur LA. 2004. Outcomes of a nutrition audit in a tertiary pediatric hospital: implications for service improvement. J. Paediatr. Child Health., 40: 295-298. DOI: http://www.ncbinlm.nih.gov, pubmed

Ousmane O. 2012. Prise en charge des enfants malnutris admis au CREN du centre médical Saint-Camille de Ouagadougou (Burkina Faso). Mémoire de maitrise, Université d'Ouagadougou, Burkina Faso, 32pp. DOI : http://www.memoireonline.com

OMS. 2000. (Organisation Mondiale de la Santé). La prise en charge de la malnutrition sévère : Manuel à l'usage des médecins et autres personnels de santé à des postes d'encadrement. PDF. https://www.who.int,nutrition,publication

Oztürk Y, Büyükgebiz B, Arslan N, Ellidokuz H. 2003. Effects of hospital stay on nutritional anthropometric data in Turkish children. J. Trop. Pediatr., 49: 189-190.

DOI: http://www.ncbinlm.nih.gov, pubmed

Pawellek I, Dokoupil K, Koletzko B. 2008. Prevalence of malnutrition in paediatric hospital patients. Clin. Nutr., 27: 72-76. DOI: http://www.ncbinlm.nih.gov, pubmed

Peláez RB. 2013. Desnutrición y enfermedad. Nutr. Hosp., 28(5): 81-88. DOI: http://www.redalyc.org,pdf

PNNS 2 (Programme National Nutrition Santé). 2006. Dénutrition une pathologie méconnue en société d'abondance PNNS1 [Internet]. PDF 
disponible sur : http://www.solidaritessante.gouv.fr/IMG/pdf/brochure_denutr ition.pdf http://www.docireps 971. canalblog.com, 2010/11/19

Rocha GA, Rocha EJ, Martins CV. 2006. The effects of hospitalization on the nutritional status of children. J. Pediatr (Rio J)., 82(1): 70-74. DOI: http://www.ncbinlm.nih.gov, pubmed

Sall M, Dankoko b, Badiane M. 1999. Results of a nutritional rehabilitation with Spirulina in Dakar. Médecine d'Afrique Noir, $\quad 46$ (3): 143-146. DOI:www.santetropicale.com/Resume/ 34604.pdf

Sall MG, Badji ML, Martin SL. 2000. Récupération nutritionnelle en milieu hospitalier régional : Le cas de l'hôpital régional de Kaolack (Sénégal). Med. Afr. Noire, 47(12): 525-527.

http://www.santetropicale.com

Schneider SM, Veyres P, Soummer AM, Jambou P, Filippi J, Van Obberghen E, Hébuterne X. 2004. Malnutrition is an independent factor associated with nosocomial infections. Br. J. Nutr., 92: 105-111. http://www.cambrige.org

Secker DJ, Jeejeebhoy KN. 2007. Subjective Global Nutritional Assessment for children. Am. J. Clin. Nutr., 85(4): 1083-1089.

http://www.ncbinlm.nih.gov, pubmed

Sermet-Gaudelus I, Poisson-Salomon AS, Colomb V, Brusset MC, Mosser F, Berrier F, Ricour C. 2000. Simple pediatric nutritional risk score to identify children at risk of malnutrition. Am. J. Clin. Nutr., 72(1): 64-70. DOI: http://www.ncbinlm.nih.gov, pubmed

Sinnaeve O, Testa J, Ablefonlin E, Ayivi B. 2006. Aspects épidémiologiques de la malnutrition infanto-juvénile à Cotonou (bénin). Med Trop, $66: 177-181$. DOI : http://www.memoireonline.com

Simpore J, Zongo F, Kabore F, Dansou D, Bere A, Pignatelli S. 2006. Nutrition rehabilitation of undernourished children utilizing Spiulina and Misola. Nutr J., 5: $3 . \quad$ DOI : http://researchgate.net, publication
Yugbare/Ouedraogo SO, Dao L, Nieni A, Togueyni L, Kisito N, Koueta F, Kam L, Ye/Ouattara D, Kaboret S. 2016. Malnutrition et hospitalisation chez les enfants de moins de cinq ans au centre hospitalier universitaire pédiatrique Charles de Gaulle de Ouagadougou (Burkina Faso). Rev. CAMES SANTE, 4(2): $\quad$ 56-62. DOI : http://www.publication.lecames.org,arti cle,view.

UNICEF. 2004. United Nations Children's Fund and World Health Organization. Unicef Supply Catalogue. http://supply.unicef.org.

Yougbaree O, Solange O, Toguyeni L, Dao LO, Nelambaye F, Nagalo K. 2017. Conduite de la diversification alimentaire du nourrisson à Ouagadougou (Burkina Faso). J Afr Pediatr Genet Med, 3: 17-22. DOI: http://www.uac.bj,pdf,jap 2.pdf

Youssao Abdou Karim I, Dahouda M, Attakpa EY, Koutinhouin GB, Ahounou GS, Toleba SS, Balogoun BS. 2013. Diversités des systèmes d'élevages de bovins de race bovine Borgou dans la zone soudanienne du Bénin. Int. J. Biol. Chem. Sci., 7(1) : 125-146. DOI : http://dx.doi.org/10.4314/ijbcs.v7i1i.11

WHO. 2006. World Health Organization. WHO Child Growth Standards: Length/Height-for-Age, Weight-forAge, Weight-for-Length, Weight-forHeight and Body Mass Index-for-Age: Methods and Development. World Health Organization, Geneva. http://www.Who.int,technical_report.

Wuehler SE, Biga A. 2011. Situational analysis of infant and young child nutrition policies and programmatic activities in Niger. Maternal and Child Nutrition, 7(1): 133-156. 